\title{
KINETIC AND STOICHIOMETRIC EVALUATION OF FREE RADICALS SCAVENGERS ACTIVITIES BASED ON DIPHENYL-PICRYL HYDRAZYYL (DPPH) CONSUMPTION
}

\author{
ANA MARIA CAMPOS, ${ }^{1}$ NICOLE DURAN, ${ }^{1}$ CAMILO LOPEZ-ALARCÓN,${ }^{2}$ AND EDUARDO LISSI ${ }^{*}$ \\ ${ }^{I}$ Facultad de Química y Biología, Universidad de Santiago de Chile (USACH) \\ ${ }^{2}$ Facultad de Química, Pontificia Universidad Católica de Chile \\ (Received: April 26, 2012 - Accepted: July 20, 2012)
}

\begin{abstract}
Kinetics and stoichiometric measurements based on the reactions of stable free radicals are among the most employed procedures in the evaluation of antioxidant activities of pure compounds and complex mixtures. In the present work it is shown that initial rates of diphenyl-picryl hydrazyl (DPPH) consumption elicited by cinnamic acid derivatives are closely related to their reactivity towards peroxyl radicals. This implies that kinetic data regarding DPPH bleaching can be useful on the evaluation of antioxidant reactivities. On the other hand, data obtained at a single (long) reaction time are unrelated to the reactivity of the antioxidants and mostly reflect the number of phenol groups present in the tested sample
\end{abstract}

Keywords: Diphenyl-picryl hydrazyl; cinnamic acids; reactivity; stoichiometry .

\section{INTRODUCTION}

Evaluation of the capacity of a given compound (free radical scavenger or antioxidant) to remove biological relevant radicals (ROS or NOS) is a matter of great interest. Similarly, much effort has been devoted to determine in a single assay the total antioxidant capacity (or activity) of complex mixtures (biological fluids, fruits, vegetables, juices, wine, etc.) and a number of reviews has been dedicated to discuss the data and the advantages and limitations of the different methodologies ${ }^{1-6}$. Among these methodologies, preferential attention has been paid to techniques based on the consumption of stable free radicals: $\mathrm{DPPH}^{7,8}$, galvinoxyl ${ }^{9}$, and ABTS derived radical cation ${ }^{10}$. These methodologies have the advantage of their experimental simplicity but suffer from important limitations. In the first place, the employed radicals are widely different than those of interest in biology, pharmacology and food chemistry. Secondly, the kinetics involved is complex ${ }^{11,12}$, making not straightforward the interpretation and the meaning of the obtained results.

Among the stable radicals most employed in the evaluation of antioxidants activity stands DPPH, whose consumption promoted by antioxidants can be easily followed by the bleaching of its absorbance at $515-520 \mathrm{~nm} \cdot{ }^{13}$ The radical removing activity of a given compound or a complex mixture is then estimated from kinetic measurements ${ }^{14,15}$ and/or for stoichiometric evaluations. In particular, it is widely employed the $\mathrm{EC}_{50}$ parameter defined as the amount of antioxidants that, at a fixed time, bleaches half of the initial DPPH absorbance. In order to consider both the reactivity and stoichiometry of the tested sample it has been proposed an index that includes the $\mathrm{EC}_{50}$ and the time required to reach the plateau at the $\mathrm{EC}_{50}$ concentration ${ }^{16,17}$. In the present work we discuss the validity of the proposed methodologies, with particular emphasis on the information that can be derived from kinetic evaluations of the rate of DPPH bleaching and the stoichiometry of the process. This is a conflicting subject and even there are not consensus regarding how to estimate the "final" DPPH concentration: 5 minutes ${ }^{18} ; 15$ minutes $^{19}, 20$ minutes $^{20} ; 30$ minutes $^{21}$, one hour $^{22}, 24$ hours $^{23}$, end of the fast decay ${ }^{24}$ or reaching a plateau ${ }^{25}$ or a steady state condition ${ }^{26,27}$.

\section{MATERIAL AND METHODS}

Cinnamic acids derivatives and DPPH (Aldrich) and ethanol (p.a. Merck) were employed as received. Stock solutions of the acids and DPPH were prepared daily in ethanol. The reaction was followed by measuring te decay of DPPH absorbance at $517 \mathrm{~nm}$ in a spectrophotometer (Unicam). A small aliquot of the cinnamic acid derivative solution was added to a cell containing DPPH $(75 \mu \mathrm{M})$ in ethanol. After a fast mixing the absorption of the sample was continuously measured (delay time $10 \mathrm{~s}$ ) during 20 minutes. All measurements were carried out at room temperature $\left(18^{\circ} \mathrm{C}\right)$.

\section{RESULTS AND DISCUSSION}

Typical results regarding DPPH consumption are given in Figure 1.

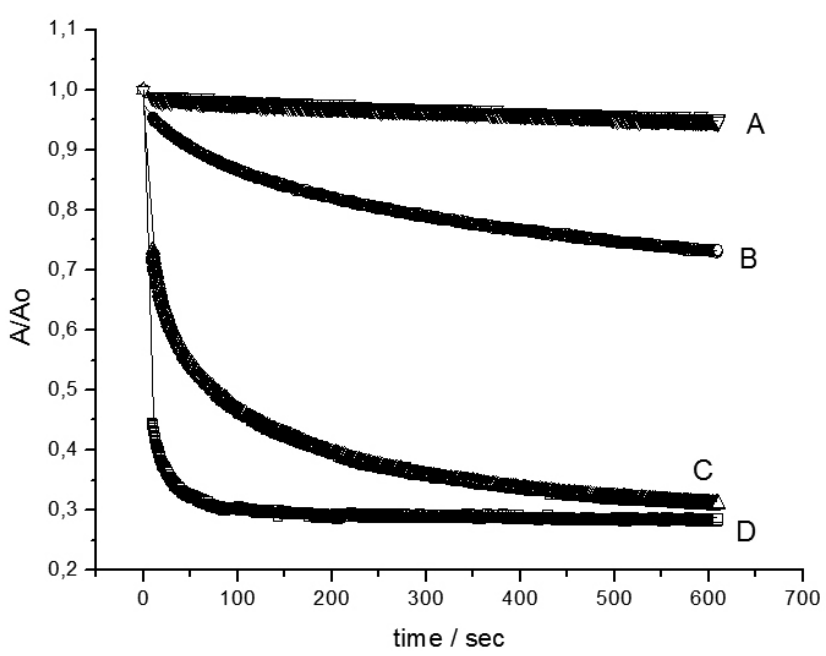

Figure 1. . Consumption of DPPH elicited by cinnamic acids addition. (A) Coumaric acid, $2.64 \mathrm{mM}$; (B) Ferulic acid, $0.028 \mathrm{mM}$; (C) Caffeic acid, $0.026 \mathrm{mM}$; (D) Sinapic acid, $0.028 \mathrm{mM}$. Solid lines correspond to the fitting to a bi-exponential decay.

Consumption of DPPH by phenols frequently does not follow a simple mono-exponential decay. In general, a fast decay is followed by a much smaller rate of consumption (See Figure 1 and Ref 19 ). In order to evaluate the rate constant of the process, we empirically fit the data to a bi-exponential decay:

$$
A_{t}=A_{\infty}+A_{1} \exp \left(-t / \Gamma_{1}\right)+A_{2} \exp \left(-t / \Gamma_{2}\right)
$$

where $\mathrm{A}$ are the amplitudes, $\Gamma_{1}$ and $\Gamma_{2}$ are the lifetimes associated to the fast and slow decays and $\mathrm{A}_{\infty}$ takes into account the unreacted DPPH at "infinite" time .

The bleaching rate, extrapolated to "zero" time, is given by

$$
-(\mathrm{dA} / \mathrm{dt})_{0}=\left(\mathrm{A}_{1} / \Gamma_{1}\right)+\left(\mathrm{A}_{2} / \Gamma_{2}\right)
$$

and a bimolecular rate constant $\left(\mathrm{k}_{\mathrm{b}}\right)$ can be defined as

$$
\mathrm{k}_{\mathrm{b}}=-(\mathrm{dA} / \mathrm{dt})_{0} /\left(\mathrm{A}_{0}[\mathrm{XOH}]\right)
$$

were $\mathrm{A}_{0}$ is the absorbance of the solution prior the antioxidant addition, corrected by the dilution associated to its incorporation, and $[\mathrm{XOH}]$ is the 
scavengers initial concentration. Average values of the bimolecular rate constants obtained are given in Table 1 .

Table 1. Second order specific rate constants for cinammic acid derivatives.

\begin{tabular}{|c|c|c|}
\hline Compound & $\begin{array}{c}\text { Range of additive } \\
\text { concentrations }(\mu \mathrm{M})\end{array}$ & $\mathbf{k}\left(10^{3}\right.$ seg $\left.^{-1} \mathrm{M}^{-1}\right)$ \\
\hline Coumaric acid & 1300 to 4000 & $0.025 \pm 0.001$ \\
\hline Ferulic acid & 28 to 532 & $52 \pm 3$ \\
\hline Synapic acid & 9 to 28 & $3470 \pm 130$ \\
\hline Caffeic acid & 11 to 52 & $2270 \pm 270$ \\
\hline
\end{tabular}

Fitting of the data to a bimolecular process is sensible to the experimental procedure employed. Working under pseudo first order conditions, Madsen et al. found a rather erratic dependence of the second order rate constant with the antioxidant concentration. ${ }^{12}$ On the other hand, Foti et al.,${ }^{24}$ reported a systematic decrease in rate constants when the substrate concentration increases. The authors attributed this peculiar effect to electron transfer processes, concluding that the reactivity of the compound towards ROS, in particular peroxyl radicals, cannot be related to the rate of their reaction with DPPH radicals. A milder position is taken by Nanjo et al. ${ }^{28}$ who concluded that reactivity with DPPH may or may not permit a clear cut definition of the radical scavenging abilities or antioxidant effects of a given compound. In the present work, where the rate constant is derived from initial consumption rates measured at different substrate concentrations, the obtained values of the second order rate constants were barely dependent on the concentration in the accessible range (see Table 1). These differences point to the complexity of the reaction and the difficulty in comparison of data obtained in different laboratories employing different experimental conditions and data treatment procedures..

The values of $\mathrm{k}$ given in Table 1 correlate with the reactivity of the cinnamic acid derivatives towards peroxyl radicals: sinapic $>$ caffeic $>$ ferulic $>$ coumaric, obtained in radical promoted oxidations of the acids ${ }^{29}$. This is emphasized by the data plotted in Fig. 2 .

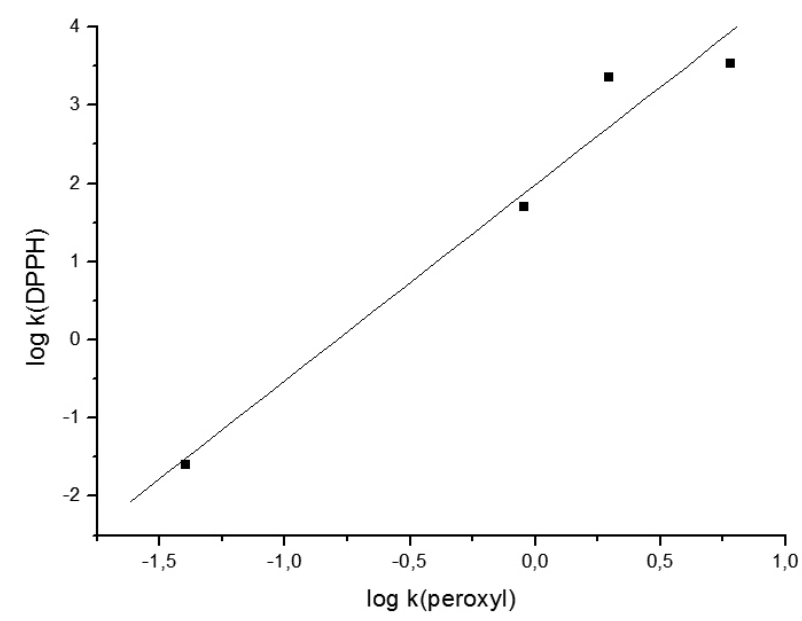

Figure 2. . Relation between the rate constants for the reaction of ROO* and DPPH radicals with cinnamic acid derivatives. Data for the reactivity of ROO* radicals adapted from Ref 41 .

This Figure shows a very good correlation between the rates constants of Table 1 for DPPH and those estimated for peroxy radicals reactions with the hydroxycinnamic acids The large slope of the plot is attributable to the smaller reactivity (and hence larger selectivity) of the DPPH radical. This allows concluding that the initial rates of DPPH consumption are related to the reactivity of the tested compound towards peroxyl radicals. If this is extrapolated to complex mixtures, the initial rates of DPPH consumption should be determined by the quantity and reactivity of the antioxidants present in the sample. However, it must be considered that the occurrence of cross reactions in complex mixtures makes much more complex the interpretation of the results ${ }^{29,30}$.
Butovic et al. ${ }^{31}$ measured the rate of reaction of several flavonoids with DPPH. Working in pseudo first order conditions (excess of flavonoid) they were able to derive the second order rate constants. The values range from 4.4 $\mathrm{x} 10^{3}$ to $0,1 \mathrm{M}^{-1} \mathrm{~s}^{-1}$ for quercetagein to apigenin, respectively. This emphasizes the great sensitivity of the kinetic process to the scavenger structure ${ }^{19}$. On the other hand, stoichiometric factors derived from titration experiments were rather similar for all the compounds considered.

Stoichiometry of the process and total number of DPPH molecules bleached by pure compounds and/or complex mixtures of antioxidants.

Frequently the antioxidant capacity of complex samples is estimated from evaluations of DPPH consumption at a fixed time (10 or 15 minutes), measured at a very long time, at the end of the initial fast reaction ${ }^{24}$, at the plateau or at the steady state ${ }^{13,17,25,32}$, or extrapolated to "infinite" time. We have chosen this last procedure in order to avoid the uncertainty associated to a visual estimation of the steady state conditions.

The data obtained can be generally well represented by a bi-exponential decay (See Figure 1). This allows the evaluation of the fraction of DPPH reacted at infinite time $\left(\mathrm{F}_{\infty}\right)$ from

$$
\left(\mathrm{F}_{\infty}\right)=\left(\mathrm{A}_{1}+\mathrm{A}_{2}\right) / \mathrm{A}_{0}
$$

It must be emphasized that in all these evaluations, and particularly those taken at very long reaction times, the relevant parameter is the amount of phenols in the complex sample and/or the number of phenolyc groups in the tested compounds, and not the reactivity of the compound towards DPPH radicals. It is then not expected a relationship between kinetic and stoichiometric evaluations. On the other hand, it is expected, and frequently found, good correlations between the number of hydroxyl groups in a compound and $\mathrm{EC}_{50}$ values $^{16}$. In complex mixtures it is frequently found a relationship between the amounts of total phenols titrated by Folin's method and the amount of DPPH molecules bleached ${ }^{33,34}$, in particular, with $\mathrm{EC}_{50}$ values $^{26,35}$. An example of this relationship is provided by data obtained on the antioxidant activity of guaya fruit extracts (Figure 3 with data from Ref 23).

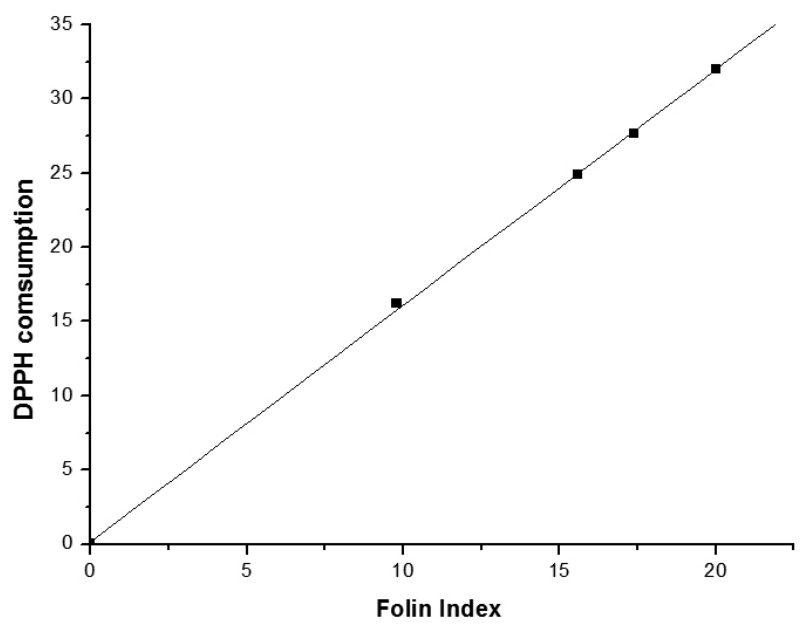

Figure 3. Correlation between DPPH consumption and total phenols determined by Folin's assay expressed in Trolox microequivalents per gram of fruit. (Adapted from Ref. 23).

This figure shows that the data correlate very well, with a slope close to one. Similar results have been reported for a large number of edible plant $\operatorname{products}^{18}(R=0.969)$, for tropical fruits ${ }^{36}(R=0.96 ; N=14)$, for herbal teas ${ }^{37}$ $(\mathrm{R}=0.93, \mathrm{~N}=10)$ and for commercially available teas in Argentina ${ }^{33}\left(\mathrm{R}^{2}=\right.$ $0.914, N=12$ ). Nevertheless, in other systems the relationship is considerably weaker ${ }^{38}$. A fair correlation between total phenols and DPPH bleaching capacity after 10 minutes of reaction has also been reported for Peruvian wines ${ }^{39}\left(\mathrm{R}^{2}\right.$ $=0.819, \mathrm{~N}=13)$, and extracts of Anacardium occidentale ${ }^{40}\left(\mathrm{R}^{2}=0.67, \mathrm{~N}=\right.$ 7). Remarkably, the stoichiometric character of these data is reinforced by the similarity between the values of total phenols and DPPH consumption. Similar 
conclusions have been reached employing the bleaching of ABTS radical cation as a measure of the additive antioxidant activity ${ }^{10}$.

The lack of kinetic information provided by consumption measurements at long times can be also established from a comparison or the number of DPPH molecules consumed by each antioxidant (n) and the time needed to reach the steady state condition. Data reported by Nenadis et al. ${ }^{32}$ stress this point (Fig 4)

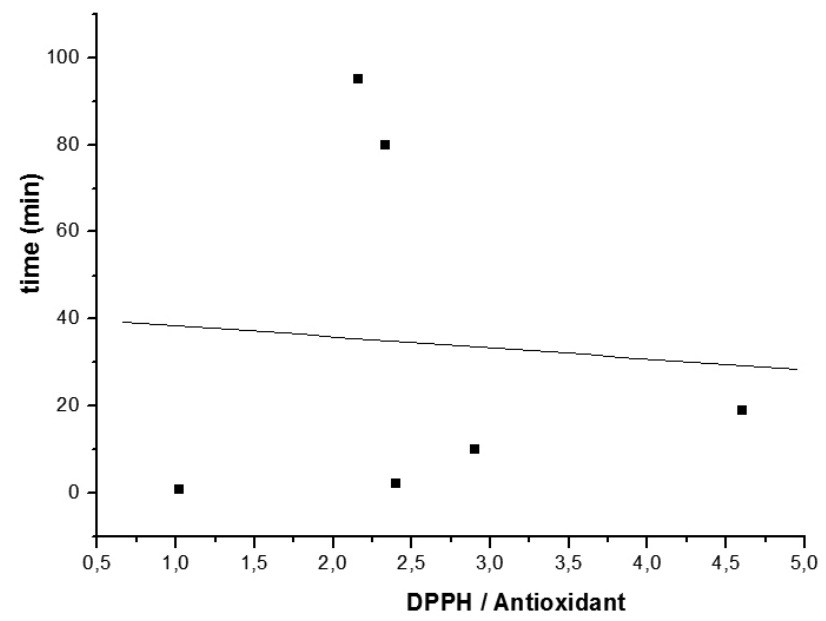

Figure 4. . Lack of relationship between the number of DPPH radical remove per antioxidant and the time required to reach the steady state at the $\mathrm{EC}_{50}$ concentration. (Data adapted from Ref. 32).

Brad-Williams et al. ${ }^{13}$ reported that the number of reduced DPPH molecules was 1.16 and 4.54 for ferulic and caffeic acids, respectively. Bondet et al. ${ }^{7}$ reported 2.8 DPPH radicals consumed by each BHT molecule incorporated into the system. The large number reported for caffeic acid is compatible with the data obtained in the present work and points to the relevance of secondary reactions $s^{38}$, particularly at long reaction times.

Table 2. Stoichiometry (n) of the process estimated for cinnamic acids.

\begin{tabular}{|l|l|c|c|}
\hline Compound & Measuring condition & $\mathrm{n}$ & Reference \\
\hline Ferulic acid & Plateau & 1.16 & 13 \\
\hline & 20 minutes reaction & 0.69 & 20 \\
\hline & End of fast step & 1.1 & 24 \\
\hline & Infinite time & 1.4 & This work \\
\hline & & & \\
\hline Sinapic acid & 20 minutes reaction & 1.34 & 20 \\
\hline & End of fast step & 1.2 & 24 \\
\hline & Infinite time & 1.9 & This work \\
\hline Caffeic acid & Plateau & 4.54 & 13 \\
\hline & 20 minutes reaction & 2.15 & 20. \\
\hline & End of fast step & 2.1 & 24 \\
\hline & Infinite time & 4.3 & This work \\
\hline
\end{tabular}

Stoichiometries obtained for the reaction of DPPH and cinnamic acids are collected in Table 2. Differences are attributable to the different techniques employed and, for slow reacting compounds (ferulic acid), to the incompleteness of the process after 20 minutes of reaction. This incomplete consumption is explainable both in terms of a slow initial step and/or to the reversibility of the secondary reactions when compounds of low reactivity are considered. ${ }^{42}$

\section{CONCLUSIONS}

The initial rate of DPPH consumption promoted by cinnamic acid derivatives correlates with their reactivities towards peroxyl radicals.

DPPH consumption, measured to a single (long) reaction time is unrelated to the reactivity of the additives. The stoichiometry of the process (n) is then almost unrelated to the reactivity of the compound considered, but is influenced by the number of hydroxyl groups and the experimental conditions employed and, in particular, by the time at which the "total" DPPH consumption is evaluated.

\section{ACKNOWLEDGEMENTS} Chile)

This work has been financed by DICYT (Universidad de Santiago de

\section{REFERENCES}

1. C. Sanchez-Moreno. Food Sci. Tech. Int. 8, 121-137 (2002)

2. R.L. Prior, X. Wu and K. Schaich. J. Agric. Food Chem. 53, 4290-4302 (2005)

3. E. Niki. and N. Noguchi. IUBMB Life 50, 323-329 (2000)

4. L.M. Magalhaes, M.A. Segundo, S. Reis, and J.L.F.C. Lima. Anal. Chim. Acta 613, 1-19 (2008)

5. E.N: Frankel and A. S. Meyer. J. Sci.Food Agric. 80, 1925-1941 (2000).

6. V. Roginsky and E. Lissi. Food Chemistry 92, 235-254 (2005)

7. V. Bondet, W. Brand-Williams and C. Berset. Lebensm.Wiss u-Technol. 30, 609-615 (1997)

8. C. Sanchez-Moreno, and J.A. Larrauri. Food Sci. Technol. Intern. 4, 391399 (1998)

9. H. Shi and E. Niki. Lipids 33, 365-370

10. P.Campodónico, E. Barbieri, M. Pizarro, C.P. Sotomayor and E.A. Lissi. Bol. Soc. Chil. Quim. 43, 281-285 (1998)

11. A.M. Campos and E.A. Lissi. Int. J. Chem. Kinetics 29, 219-224 (1997)

12. H.L. Madsen, C.M. Andersen, L.V. Jorgensen and L.H. Skibsted. Eur. Food Res. Technol. 211, 240-246 (2000)

13. W. Brand-Williams, M.E. Cuvalier and C. Berset. Lebsen-Wiss.uTechnol. 28, 25-30 (1995)

14. P. Stratil, B. Klejdus and V. Kuban. J. Agric. Food Chem. 54, 607-616 (2006)

15. J. Imai, N. Ede, S. Nagae, T. Morigguchi, H. Matsuura and Y. Itakura Planta Med. 60, 417-420 (1984).

16. N. Nenadis, O. Lazaridou and M.Z. Tsimidou. J. Agric. Food Chem.55, 5452-5460 (2007)

17. C. Sanchez-Moreno, J.A. Larrauri, and F. Saura-Calixto. J. Sci. Food Agric. 76, 270-276 (1998)

18. T. Katsube, H. Tabata, Y. Ohta, Y. Yamasaki, E. Anuurad, K. Shiwaku, and Y. Yamane. J. Agric. Food Chem. 52, 2391-1396 (2004)

19. P. Goupy, C. Dufour, M. Loonis and O. Dangles. J. Agric Food Chem. 51, 615-622 (2003)

20. P. Cos, P. Rajan, I. Vedernikova, M. Calomme, L. Pieters, A. Vlietinck, K. Agustyns, A. Haemers, and D. Vanden Berghe. Free Rad. Res. 36, 711-716 (2002).

21. S.H.Wong, L.P. Leong, and J. H. W. Koh. Food Chem. 99, 775-783 (2006)

22. B. Escuder, R. Torres, E. Lissi, C. Labbe and F. Faini. Nat.Prod.Letters 16, 277-281 (2002).

23. K. Thaipong, U. Boonprakob, K. Crosby, L Cisneros-Zevallos, D. H. Byrne. J. Food Comp. Analysis. 19, 669-675 (2006)

24. M.C. Foti, C. Daquino and C. Gerac. J. Org. Chem. 69, 2309-2314 (2004)

25. N. Saint-Cricq de Gaulejac, C. Provost and N. Vivas. J. Agric. Food Chem. 47, 425-431 (1999)

26. A. Jimenez-Escrig. I.Jimenez-Jimenez, R. Pulido and F. Saura-Calixto. J. Sci.Food Agric .81, 630-634 (2001)

27. N. Nenadis, O. Lazaridou, and M.Z. Tsimidou. J. Agric. Food Chem. 55, 5452-5460 (2007).

28. F. Nanjo, K. Goto, R. Seto, M. Suzuki, M. Sakai and Y. Hara. Free Rad. Biol. Med. 21, 895-902 (1996)

29. C. López-Alarcón, A. Aspée and E. Lissi. Free. Rad. Res. 41, 1189-1194 (2007)

30. A. Blauz, T. Pilaszek, A. Grzelak, A. Dragan, and G. Bartosz. Food. Chem. Toxicol. 46, 2365-2368 (2008) 
31. V. Butovic, L. Klasinc and W. Bors. J. Agric. Food Chem. 52, 2816-2820 (2004)

32. N. Nenadis, S- Bpuñe. E. G. Bakalbassis and M. Tsimidou. Am. Oil Chem. Soc. 80, 451-458 (2003)

33. C. Anesini, G.E. Ferraro and R. Filip. J. Agric. Food Chem. 56, $9225-$ $9229(2008)$

34. Y.Y. Lim, T.T. Lim, and J.J. Tee. Food Chem.103, 1003-1008 (2007)

35. C.B. de Oliveira, L. N. Comunello, A. Lunardelli, R.H. Amaral, M. G. S Pires, G. L. da Silva, V. Manfredini, C. R. Vargas, S. C.B. Gnoatto, J. R. de Oliveira and G . Gosmann. Molecules 17, 1113-1123 (2012)

36. K. Mahattanatawee, J. A. Manthey, G. Luzio, S.T. Talcott, K. Goodner and E. A. Baldwin. J. Agric. Food Chem. 54, $7355-7363$ (2006)
37. A. Buyukbalci and S. Nehir El. Plant Food Hum. Nutr. 63, 27-33 (2008)

38. C. López-Alarcón, A. Aspèe and E.A. Lissi. J. Chil. Chem. Soc. 53, 17181719 (2009)

39. A.M. Muñoz, A. F. Fernández, F. Ramos and C. Alvarado-Ortiz. Rev. Soc. Quim. Perú 73, 30-40 (2007)

40. N.M. Mokhtar, K. M. , M.. Kanthimathi and A.A. Aziz. Malasian J. Biochem. Mol. Biol. 16, 16-21 (2008)

41. F. Natella, M. Nardini, M. Di Felice and C. Scaccini. J. Agric. Food Chem. 47, 1453-1459 (1999)

42. E.A. Lissi, B. Modak, R. Torres, J. Escobar and A. Urzua. Free Rad. Res. 30, 471-477 (1999). 